\title{
Alveolar Soft Part Sarcoma of the Uterine Cervix
}

\author{
Andrew Flint, M.D., 'Paul W. Gikas, M.D., and James A. Roberts, M.D.* \\ Department of Pathology, and Division of Gynecologic Oncology, ${ }^{*}$ Department of Obstetrics and \\ Gynecology, The University of Michigan, Ann Arbor, Michigan 48109
}

Received January 22, 1985

\begin{abstract}
Alveolar soft part sarcoma is a histologically distinctive neoplasm of uncertain histogenesis. Since its initial description in 1952, more than 200 cases have been reported. The extremities are most often the sites of involvement; the tongue, bones, and the orbit have been less commonly involved. The present paper describes a case of alveolar soft part sarcoma which was present only within the uterine cervix of a 37-year-old woman. Histologically, the tumor cells were arranged in the characteristic alveolar pattern; diagnostic PAS-positive diastase-resistant needle-shaped crystals were observed within the cytoplasm of the tumor cells. After the initial biopsy, the patient underwent a radical hysterectomy and pelvic lymph node dissection. Although no residual tumor was found within the cervix, a microscopic focus of tumor was detected in an obturator lymph node. The patient is at present clinically free of disease. (c) 1985 Academic Press, Inc.
\end{abstract}

\section{INTRODUCTION}

Sarcomas are the least common primary neoplasms which may arise in the cervix. Rotmensch and colleagues [1], in a recent review, stated that these neoplasms account for less than $1 \%$ of all malignant cervical tumors. According to published reports, leiomyosarcoma and stromal sarcomas (homologous and heterologous) appear to be the most common forms. In 1952, Christopherson et al. [2] reported 12 patients who had an unusual, histologically distinctive soft tissue tumor which was characterized by an indolent but relentless clinical course. Christopherson termed this unique tumor an alveolar soft part sarcoma (ASPS); more than 200 cases have been subsequently reported in nearly 40 publications. So far as can be ascertained, there is no literature concerning the occurrence of ASPS in the uterine cervix. The present paper describes the clinical and pathologic features of an alveolar soft part sarcoma which was present only within the cervix.

\section{CASE REPORT}

A previously healthy 37-year-old woman, gravida II, para II, was admitted for evaluation of intermenstrual bleeding. A pelvic examination revealed a $2-\mathrm{cm}$

${ }^{1}$ To whom correspondence should be addressed: Department of Pathology, Box MO45, 1335 Catherine Road, Ann Arbor, Mich. 48109. 
polypoid mass which partially replaced the anterior portion of the cervix. The uterus was of normal size and texture; neither adnexal masses nor parametrial thickening were present. The remainder of the patient's evaluation was normal. A biopsy specimen of the mass was interpreted as a mesenchymal neoplasm, although an adenosquamous carcinoma was also considered by one observer. $A$ radical hysterectomy, bilateral pelvic lymph node and paraaortic lymph node dissections were performed. Residual tumor was not found in the cervix; a microscopic focus of tumor was detected in one obturator lymph node. The cervical biopsy specimen was further studied by electron microscopy; based upon the ultrastructural findings and a reexamination of the light microscopic features, a diagnosis of alveolar soft part sarcoma was rendered. A postoperative abdominal CAT scan as well as an intravenous pyelogram were performed and reported as normal. The patient made an uneventful recovery and was discharged 9 days after admission.

\section{MATERIALS AND METHODS}

Formalin-fixed biopsy material was processed routinely and stained with haematoxylin and eosin, periodic acid-Schiff (PAS) with and without diastase digestion, Alcian blue, and mucicarmine. Small pieces of fresh tumor tissue were fixed in $2 \%$ cacodylate-buffered glutaraldehyde and postfixed in similarly buffered $1 \%$ osmium tetroxide. Ultrathin sections cut from Epon-embedded tissue were stained with uranyl acetate and lead citrate. The sections were examined with a Zeiss EM 109 electron microscope.

\section{MORPHOLOGIC FINDINGS}

As observed within the excisional biopsy, the sharply circumscribed tumor was located beneath the surface mucosa (Fig. 1). The tumor cells were relatively uniform in appearance and exhibited a pseudoalveolar or organoid architectural pattern (Fig. 2). The nests of cells were separated by thin fibrous septa which contained delicate endothelial-lined vascular channels. The cells were large and often polyhedral with finely granular, acidophilic cytoplasm, and distinct cell boundaries. The abundant cytoplasm was eosinophilic and finely granular. In addition, the cytoplasm was intensely PAS positive and diastase resistant, but did not stain with the mucicarmine stain. Cytoplasmic staining by Alcian blue was equivocal. The round to oval nuclei were eccentric and often contained a single prominent nucleolus. The chromatin was uniformly arranged and nuclear pleomorphism was infrequently observed. Mitotic figures and binucleated cells were rarely present. Necrosis was not observed. Cytoplasmic, fine, needle-shaped PAS-positive crystals were present focally (Fig. 3).

Ultrastructurally, the tumor cells contained numerous mitochondria and fragmented rough endoplasmic reticula. Many of the endoplasmic cisternae were dilated into polyhedral shapes; crystalline structures were not, however, contained within them. 


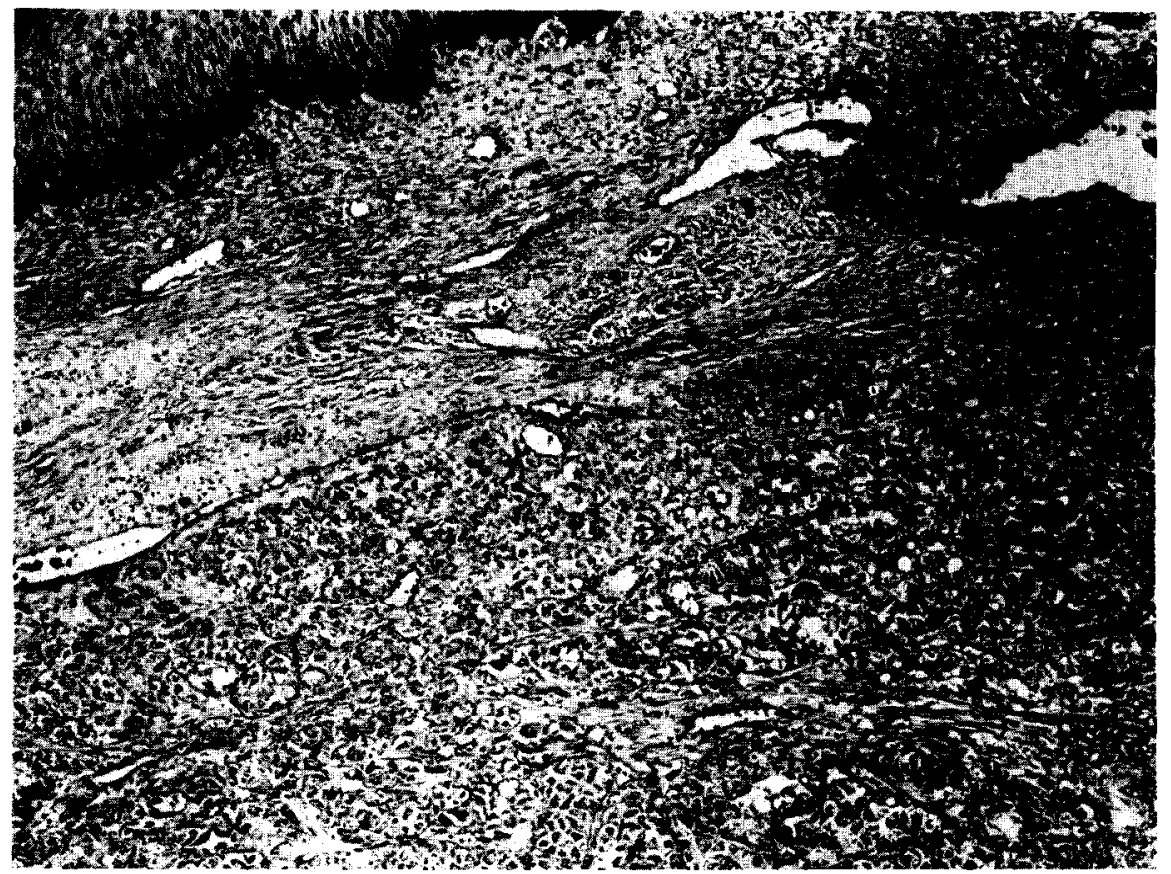

Fig. 1. The circumscribed border of the tumor is seen in this field. The adjacent cervical stroma appears compressed. (Hematoxylin and Eosin, $\times 50$ ).

\section{DISCUSSION}

Most reported cases of alveolar soft part sarcoma occur in young adults and arise within the soft tissue of the extremities. Other tumor sites have included the orbit, tongue, and bones. It is an uncommon tumor, accounting for only $1.1 \%$ of all soft tissue sarcomas [3]. Clinically, the tumor may be present as a palpable nodule for months to years before medical attention is sought. While local recurrence is uncommon, distant metastases frequently occur, often many years after the initial excision. The lungs, long bones, and brain are the most common sites of metastases. All reported deaths, with few exceptions, have been due to metastases [4].

While highly characteristic and virtually diagnostic, the PAS-positive, diastaseresistant crystals are not observed in every case; in fact, these structures werc found in only 11 of the 50 cases reported by Lieberman and his co-workers [5]. A nonchromaffin paraganglioma, an alveolar-type rhabdomyosarcoma, and metastatic renal cell carcinoma may all histologically resemble ASPS. In paragangliomas, the vascular supply is more prominent. The tumor cells vary in size, and the cytoplasm is characteristically PAS negative. With regard to rhabdomyosarcoma, the predominant cell is round and has scanty eosinophilic cytoplasm which is occasionally vacuolated. Tadpole and racquet-shaped cells are often present. Metastatic renal cell carcinoma may closely resemble ASPS; however, the positive cytoplasmic PAS staining is removed by prior diastase digestion. In 


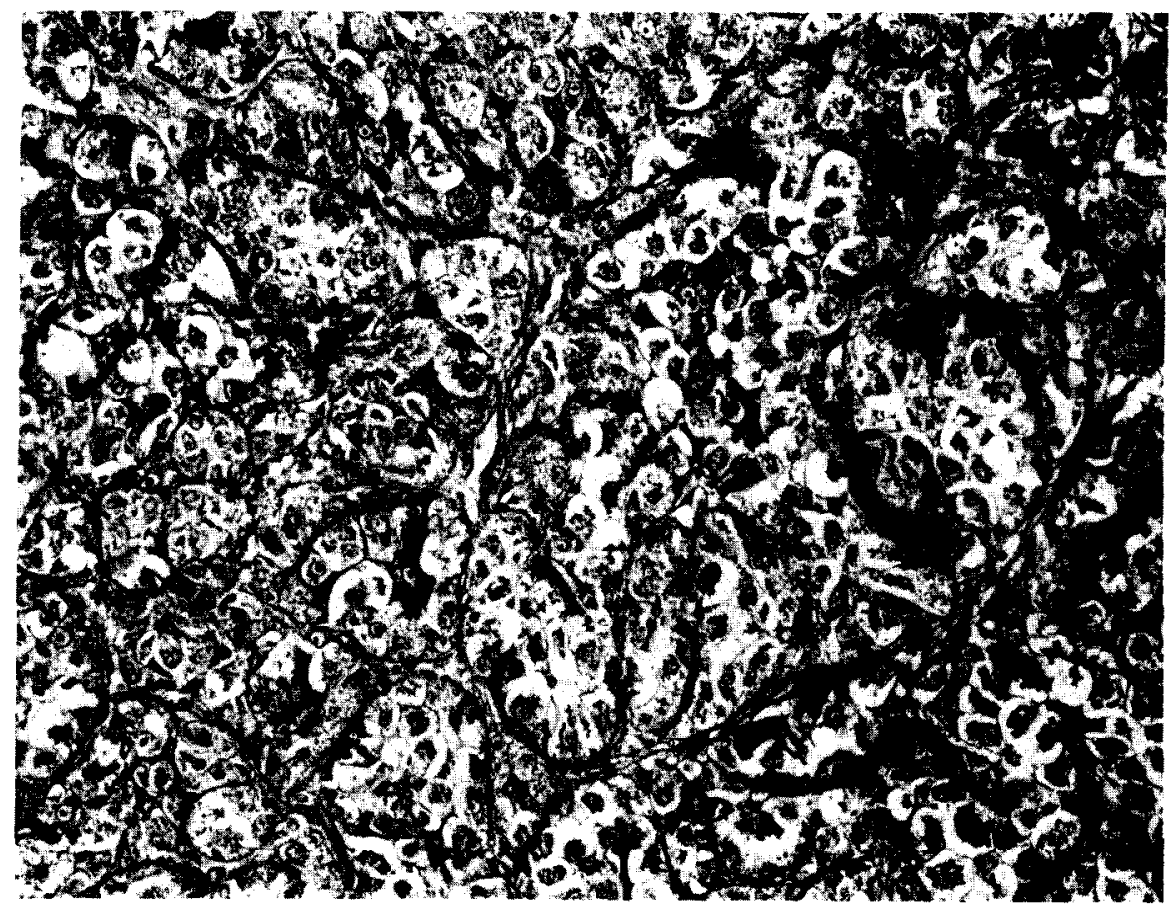

FIG. 2. Groups of tumor cells are outlined by delicate connective tissue septae, forming the characteristic "alveolar" pattern. The tumor cells have abundant cytoplasm. (Hematoxylin and Eosin, $\times 312$ ).

addition, the needle-shaped crystals are absent in all of these tumors [6,7]. The histogenesis of ASPS remains controversial; origins from neural tissue, striated muscle tissue, and paraganglia have all been postulated [8].

There do not appear to be any other reported examples of ASPS of the uterine cervix. One vulvar [3] and two vaginal [4,9] alveolar soft part sarcomas comprise the only other gynecologic examples of the tumor. Because of the extreme rarity of the tumor, there is no adequate information regarding treatment. Nevertheless, surgery probably provides the best chance of controlling the disease and curing the patient [8]; the relatively circumscribed nature of the lesion helps to ensure its complete removal. Shen et al., in reviewing the available literature [3], recommended wide excision if the tumor is localized. In view of autopsy studies which indicate that nodal metastases do occur in as many as $30 \%$ of cases, the authors also recommended dissection of regional lymph nodes. Local radiation is indicated for the tumor not amenable to excision with a wide margin. Postoperative radiaton may reduce local recurrence; however, distant metastasis remains frequent. The role of chemotherapy has not yet been defined. Lifelong clincal follow-up is important in view of late metastases; Brodsky and colleagues [10] cited three patients in whom metastases were found after a 15-year disease-free interval.

\section{ACKNOWLEDGMENTS}

We thank Dr. Tariq M. Murad (Northwestern University), Dr. Michael R. Hendrickson (Stanford University), and Dr. Franz Enzinger (Armed Forces Institute of Pathology) for reviewing the pathologic material. 


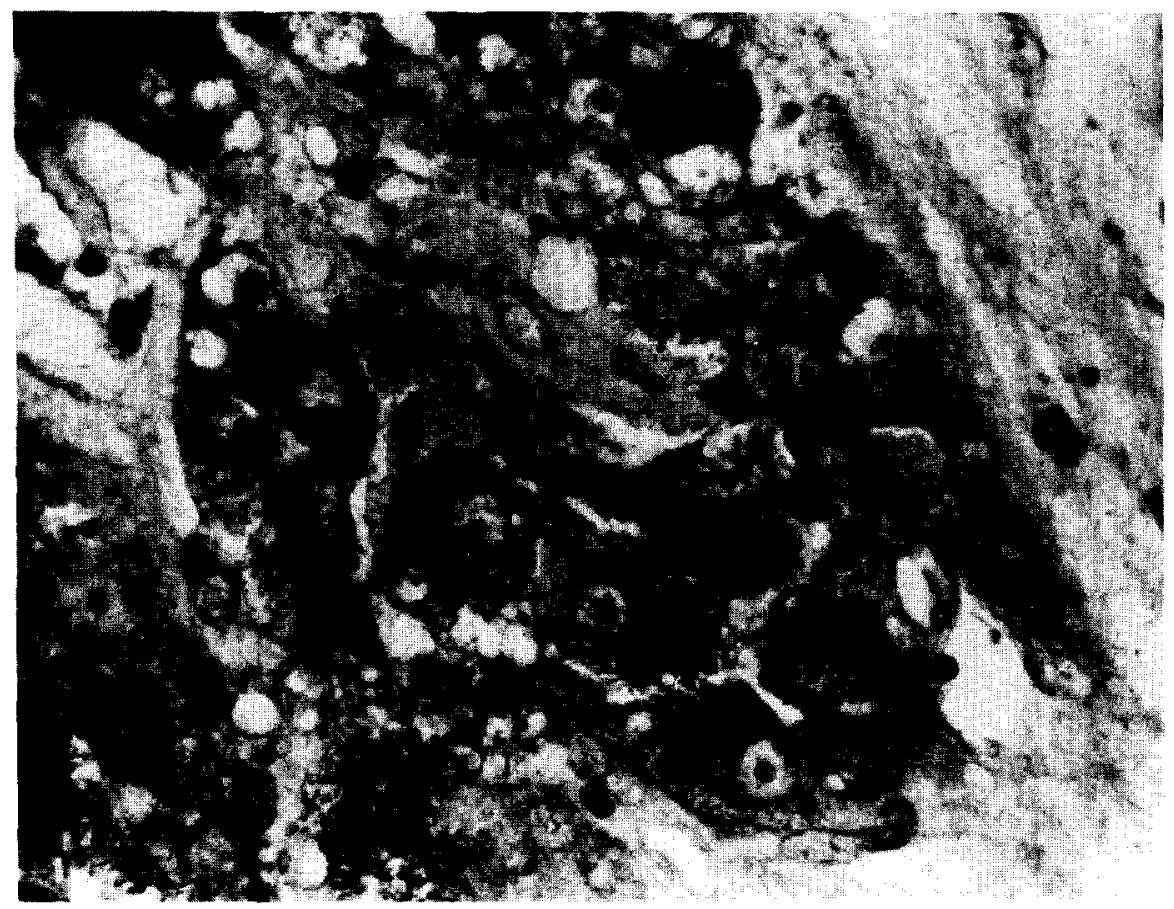

Fig. 3. Needle-shaped cytoplasmic crystalline structures are present in the center of the field. Relatively few collections of crystals were present in the histologic sections. (Periodic acid-Schiff stain, $\times 500)$.

\section{REFERENCES}

1. Rotmensch, J., Rosenshein, N. B., and Woodruff, J. D. Cervical sarcoma: A review, Obstet. Gynecol. Surv. 38, 456-460 (1983).

2. Christopherson, W. M., Foote, F. W., and Stewart, F. W. Alveolar soft part sarcomas: Structurally characteristic tumor of uncertain histogenesis, Cancer 5, 100-111 (1952).

3. Shen, J-T., D'Ablaing, G., and Morrow, C. P. Alveolar soft part sarcoma of the vulva: Report of first case and review of literature, Gynecol. Oncol. 13, 120-128 (1982).

4. Chapman, G. W., Benda, J., and Williams, T. Alveolar soft-part sarcoma of the vagina, Gynecol. Oncol. 18, 125-129 (1984).

5. Lieberman, P. H., Foote, Jr., F. W. Stewart, F. W., and Berg, J. W. Alveolar soft-part sarcoma, J. Amer. Med. Assoc. 198, 1047-1051 (1966).

6. Hamperl, H., and Lattes, R. Study of argyrophilia of nonchromaffin paragangliomas and granular cell myoblastomas, Cancer 10, 408-413 (1957).

7. Marshall, R. B., and Horn, R. C., Jr. Nonchromaffin paraganglioma. A comparative study, Cancer 14, 779-787 (1961).

8. Font, R. L., Jurco, S., and Zimmerman, L. E. Alveolar soft-part sarcoma of the orbit: A clinicopathologic analysis of seventeen cases and a review of the literature, Hum. Pathol. 13, 569-579 (1982).

9. Kasai, K., Yoshida, Y., and Okumura, M. Alveolar soft part sarcoma in the vagina: Clinical features and morphology, Gynecol. Oncol. 9, 227-236 (1980).

10. Brodsky, A. E., Dennis, M. D., and Sassard, W. R. Alveolar soft-part sarcoma, J. Bone Joint Surg. 65-A, 841-842 (1983). 